\title{
əDesigning Multimodel Applications with Surrogate Forecast Systems
}

\author{
LEONARD A. SMITH \\ Centre for the Analysis of Time Series, London School of Economics, London, \\ and Pembroke College, Oxford, United Kingdom \\ HAILIANG DU \\ Centre for the Analysis of Time Series, London School of Economics, London, \\ and Department of Mathematical Sciences, Durham University, Durham, United Kingdom \\ SARAH HIGGINS \\ Centre for the Analysis of Time Series, London School of Economics, London, United Kingdom
}

(Manuscript received 22 March 2019, in final form 31 October 2019)

\begin{abstract}
Probabilistic forecasting is common in a wide variety of fields including geoscience, social science, and finance. It is sometimes the case that one has multiple probability forecasts for the same target. How is the information in these multiple nonlinear forecast systems best "combined"? Assuming stationarity, in the limit of a very large forecast-outcome archive, each model-based probability density function can be weighted to form a "multimodel forecast" that will, in expectation, provide at least as much information as the most informative single model forecast system. If one of the forecast systems yields a probability distribution that reflects the distribution from which the outcome will be drawn, Bayesian model averaging will identify this forecast system as the preferred system in the limit as the number of forecast-outcome pairs goes to infinity. In many applications, like those of seasonal weather forecasting, data are precious; the archive is often limited to fewer than $2^{6}$ entries. In addition, no perfect model is in hand. It is shown that in this case forming a single "multimodel probabilistic forecast" can be expected to prove misleading. These issues are investigated in the surrogate model (here a forecast system) regime, where using probabilistic forecasts of a simple mathematical system allows many limiting behaviors of forecast systems to be quantified and compared with those under more realistic conditions.
\end{abstract}

\section{Introduction}

Forecasters are often faced with an ensemble of model simulations that are to be incorporated into quantitative forecast system and presented as a probabilistic forecast. Indeed, ensembles of initial conditions have been operational in weather centers in both the United States (Kirtman et al. 2014) and Europe (Palmer et al. 2004; Weisheimer et al. 2009) since the early 1990s, and there is a significant literature on their interpretation (Raftery et al. 2005; Hoeting et al. 1999; Roulston and Smith 2003; Wang and Bishop 2005;

๑ Denotes content that is immediately available upon publication as open access.

Corresponding author: Hailiang Du, hailiang.du@durham.ac.uk
Wilks 2006; Wilks and Hamill 2007). There is significantly less work on the design and interpretation of ensembles over model structures, although such ensembles are formed on weather (TIGGE; Bougeault et al. 2010), seasonal (ENSEMBLES; Weisheimer et al. 2009) and climate (CMIP5; Taylor et al. 2012) forecast lead times (expansions of acronyms can be found online at https://www.ametsoc.org/PubsAcronymList). This paper focuses on the interpretation of multimodel ensembles in situations in which data are precious, that is, in which the forecast-outcome archive is relatively small. Archives for seasonal forecasts fall into this category, typically limited to between 32 and 64 forecast-outcome pairs. ${ }^{1}$ At times, the forecaster has only an "ensemble of

\footnotetext{
${ }^{1}$ The observational data available for initialization and evaluation of the forecasts are very different before the satellite era.
} 
convenience" composed by collecting forecasts made by various groups for various purposes. Alternatively, multimodel ensembles could be formed in collaboration using an agreed experimental design. This paper was inspired by the ENSEMBLES project (Weisheimer et al. 2009), in which seven seasonal models were run in concert, with nine initial condition simulations under each model (Hewitt and Griggs 2004). Small-archive parameters ${ }^{2}$ (SAP) forecast systems are contrasted with largearchive parameters (LAP) forecast systems using the lessons learned in experimental design based on the results originally reported by Higgins (2015).

We adopt the surrogate model context, taking relatively simple models of a chaotic dynamical system, then contrasting combinations of model to gain insight in how to build and test multimodel ensembles in a context where the data are not precious and a "perfect model" (the system) is known. In this context a robust experimental design can be worked out. There is, of course, an informal subjective judgement regarding how closely the consideration in the surrogate experiments map back into the real-world experiment. This is illustrated using a relatively simple chaotic dynamical system. Specifically, the challenges posed when evaluation data are precious are illustrated by forecasting a simple one-dimensional system using four imperfect models. A variety of ensemble forecast system designs are considered: the selection of parameters and the relative value of "more" ensemble members from the "best" model are discussed. This consideration is addressed in a new generalization of the surrogate modeling framework (Smith 1992 and references therein); it is effectively a "surrogate forecasting system" approach, of value when practical constructions rule out the use of the actual forecast system of interest, as is often the case. In the large forecast-archive limit, the selection of model weights is shown to be straightforward and the results are robust as expected; when a unique set of weights are not well defined, the results remain robust in terms of predictive performance. It is shown that when the forecast-outcome archive is nontrivial but small, as it is in seasonal forecasting, uncertainty in model weights is large. The parameters of the individual model probabilistic forecasts vary widely between realizations in the SAP case; they do not do so in the LAP case. This does not guarantee that the forecast skill of SAP is significantly inferior to that of LAP, but it is shown that in this case the

\footnotetext{
${ }^{2}$ Here the parameters refer to the parameters involved in transforming the multimodel ensemble into the predictive distribution-for example, the model weights, dressing, and blending parameters (see appendix), and they are estimated from an archive that is sometimes large and sometimes small.
}

SAP forecast systems are significantly (several bits) less skillful. The goal of this paper is to refocus attention on this issue, not to claim to have resolved it. When evaluating models that push the limits of computational abilities of the day, one is forced to use systems simpler than those targeted by operational models to investigate ensemble forecasting. And whenever simplified models are employed, there is a question as to whether the results hold in larger (imperfect) models. This question of "even in or only in" was discussed in Gilmour and Smith (1997).

In turning to the question of forming a multimodel forecast system, it is shown that 1) the model weights assigned given SAP are significantly inferior to those under LAP (and, of course, to those using ideal weights), 2) estimating the best model in SAP is problematic when the models have similar skill, and 3) multimodel "out of sample" performance is often degraded because of the assignment of low (zero) weights to useful models. Potential approaches to this challenge (other than waiting for decades) are discussed. It is not possible, given the current archive, to establish the extent to which these results are relevant. The aim of the paper can only be to suggest a more general experimental design in operational studies that would identify or rule out the concerns quantified above. The paper merely raises a concern to which no exceptions are known, it does not attempt (nor could any paper today succeed) in showing this clear and present challenge to multimodel forecasting that dominates seasonal (or other) operational forecasts. It does, by considering well designed surrogate forecasting systems, provide insight into challenges likely to be faced by any multimodel forecast system of a design similar to the real forecast system of interest. ${ }^{3}$

\footnotetext{
${ }^{3}$ After reading this section, a reviewer asks whether these results are relevant to readers of Monthly Weather Review. Consider the related question: what evidence is in hand that any approach is robust in operational models? Detailed questions of how large an ensemble should be or how a multimodel should be weighted [or even constructed (Du and Smith 2017)] cannot be explored with operational models because of the extreme computational cost of such an evaluation. One could not evaluate, say, Fig. 13 using operational models. The aim of surrogate modeling is to address such questions and demonstrate the robustness of the results for simpler target systems. The weakness of surrogate forecast systems is interpreting their relation of these results to those of operational systems of interest. The alternative is to have no well quantified and evaluated insight into the robustness at all. Were the results of Hide (1958) and Read (1992) useful to numerical weather forecasting? Were the many systems of mathematical equations constructed by Lorenz $(1963,1995)$ useful? Were the circuit studies on ensemble size by Machete and Smith (2016) useful? Surrogate forecast systems can aid in the design of operational test beds and support their findings. The answer in our particular case appears to be that they are relevant.
} 


\section{From ensemble(s) to predictive distribution}

The ENSEMBLES project considered seasonal forecasts from seven different models; an initial condition ensemble of nine members was made for each model and launched four times per year (in February, May, August, and November). The maximum lead time was seven months, except for the November launch, which was extended to 14 months. Details of the project can be found in Alessandri et al. (2011), Doblas-Reyes et al. (2010), Weigel et al. (2008), Hewitt and Griggs (2004), Weisheimer et al. (2009), and Smith et al. (2015).

The models are not exchangeable in terms of the performance of their probabilistic forecasts. Construction of predictive functions via kernel dressing and blending with climatology [see Bröcker and Smith (2008) and the appendix for mathematical details] for each initial condition ensemble of simulations is discussed in Smith et al. (2015) (under various levels of cross validation). Note that kernel dressing is not kernel density estimation (Silverman 1986); asked to suggest a reference that clarifies this common confusion of the two procedures, Silverman replied "As for anything in print, this is like asking for something in print that says the earth is round rather than flat." (B. Silverman 2018, personal communication). Kernel dressing does aim to reproduce the imperfect-model distribution from which it was drawn; kernel density estimation always and only attempts to reproduce the distribution from which the ensemble members were drawn. Throughout the current paper, skill is quantified with I. J. Good's logarithmic score (Good 1952; Roulston and Smith 2002); this score is sometimes (and in this paper) referred to as "ignorance" (Roulston and Smith 2002). As noted in Smith et al. (2015) and Du and Smith (2017), ignorance is the only proper and local score for continuous variables (Bernardo 1979; Raftery et al. 2005; Bröcker and Smith 2007), and it is defined by

$$
S[p(y), Y]=-\log _{2}[p(Y)],
$$

where $Y$ is the outcome and $p(Y)$ is the probability of the outcome $Y$. In practice, given $K$ forecast-outcome pairs $\left\{\left(p_{i}, Y_{i}\right) \mid i=1, \ldots, K\right\}$, the empirical average ignorance score of a forecast system is then

$$
S_{E}[p(y), Y]=\frac{1}{K} \sum_{i=1}^{K}-\log _{2}\left[p_{i}\left(Y_{i}\right)\right]
$$

In practice, the skill of a forecast system can be reflected by the ignorance of the forecast system relative a reference forecast $p_{\text {ref: }}$ :

$$
S_{\text {rel }}[p(y), Y]=\frac{1}{K} \sum_{i=1}^{K}-\log _{2}\left\{\left[p_{i}\left(Y_{i}\right)\right] / p_{\text {ref }}\left(Y_{i}\right)\right\} .
$$

The climatological forecast ("climatology") is a commonly used reference forecast in meteorology.

\section{Simple chaotic system models pair}

Without any suggestion that probabilistic forecasting of a one-dimensional chaotic map reflects the complexity or the dynamics of seasonal forecasting of the Earth system, this paper draws parallels. Parallels between challenges to probabilistic forecasting of scalar outcomes using multiple models with different structural model errors and a small forecast-outcome archive in low-dimensional systems and those in high-dimensional systems. These challenges occur both in low-dimensional systems and in high-dimensional systems. Whether or not suggestions inspired by the low-dimensional case below generalize to high-dimensional cases (or other low-dimensional cases, for that matter), would have to be evaluated on a case-bycase basis. The argument below is that the challenges themselves can be expected in high-dimensional cases, leading to the suggestion that they should be considered in the design of all multimodel forecast experiments.

The system to be forecast throughout this paper is the Moran-Ricker map (Moran 1950; Ricker 1954) given in Eq. (4) below. Selection of a simple, mathematically defined system allows the option of examining the challenges of a small forecast-outcome archive in the context of results based on very large archives. This is rarely possible for a physical system (see, however, Machete 2007; Smith et al. 2015). In this section the mathematical structure of the system and four imperfect models of it are specified. The specific structure of these models reflects a refined experimental design in light of the results of Higgins (2015).

Let $\tilde{x}_{t}$ be the state of the Moran-Ricker map at time $t \in \mathbb{Z}$. The evolution of the system state $\tilde{x}$ is given by

$$
\tilde{x}_{t+1}=\tilde{x}_{t} \exp \left[\lambda\left(1-\tilde{x}_{t}\right)\right] .
$$

In the experiments presented in this paper we use $\lambda=3$, where the system is somewhat "less chaotic," rather than using the value adopted in Sprott (2003) [Fig. 1 shows the Lyapunov exponent as a function of system parameter $\lambda$ (Glendinning and Smith 2013)] in order to ease the construction of models with comparable forecast skill. We define the observation at time $t$ to be $s_{t}=\tilde{x}_{t}+\eta_{t}$, where the observational noise $\eta_{t}$ is independent and normally distributed $\left[\eta_{t} \sim N\left(0, \sigma_{\text {noise }}^{2}\right)\right] .{ }^{4}$

\footnotetext{
${ }^{4}$ Observations are restricted to positive values.
} 

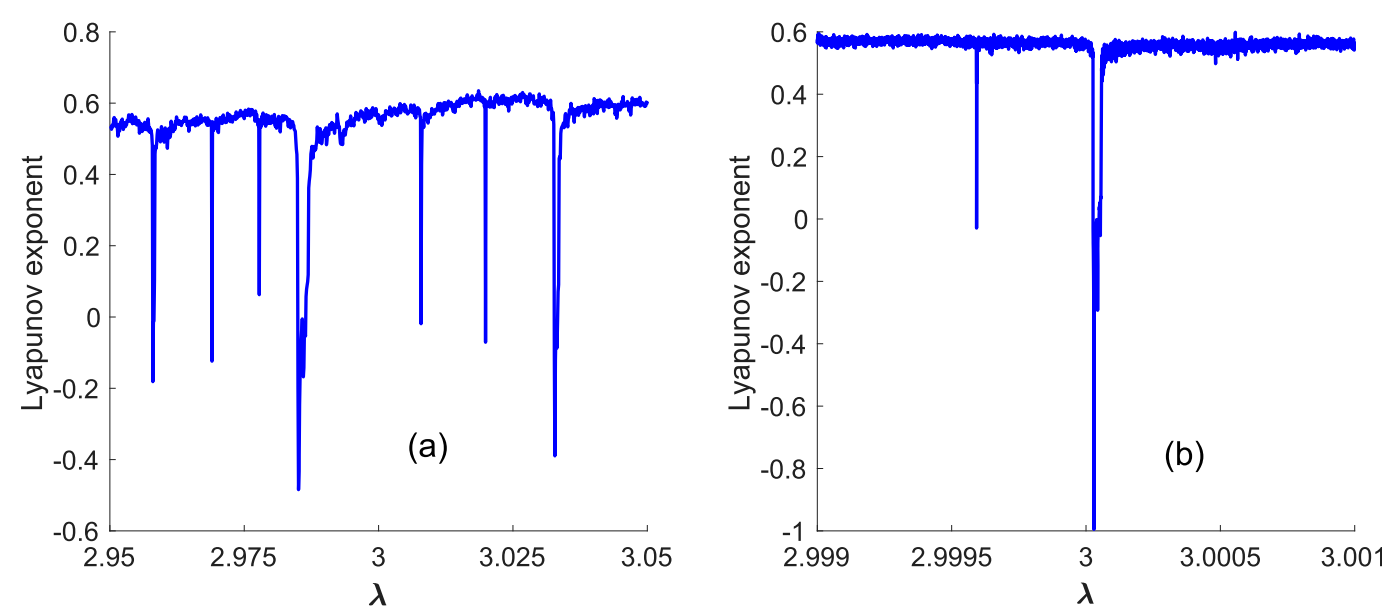

FIG. 1. Estimates of the global Lyapunov exponent plotted as a function of $\lambda$ : (a) 4096 values of $\lambda$ uniformly random sampled between 2.95 and 3.05 and (b) 4096 values of $\lambda$ uniformly random sampled between 2.999 and 3.001.

Four one-dimensional deterministic models are constructed, each one being an imperfect model of the Moran-Ricker system. In the experiments presented here, the focus is on designing a multimodel ensemble scheme and effective parameter selection for producing predictive distribution from multiple models. Therefore the imperfect models as well as their parameter values are fixed. These four models share the same state space as the system, and the observations are complete. Note in practice, it is almost always the case that the model state $x$ lies in a different space from the system state $\tilde{x}$. The models are as follows:

Model I, $G_{1}(x)$, is built by first expanding the exponential term in Eq. (4) to the 12th order:

$$
\begin{aligned}
x_{t+1}= & x\left\{1+\lambda(1-x)+\frac{1}{2 !}[\lambda(1-x)]^{2}+\cdots\right. \\
& \left.+\frac{1}{12 !}[\lambda(1-x)]^{12}\right\} .
\end{aligned}
$$

The coefficient of each polynomial term is then truncated at the third decimal place:

$$
\begin{aligned}
x_{t+1}= & x\left[1+3(1-x)+4.5(1-x)^{2}+\cdots\right. \\
& \left.+0.004(1-x)^{11}+0.001(1-x)^{12}\right] .
\end{aligned}
$$

Model II, $G_{2}(x)$, is derived by first taking the logarithm of Eq. (4) and expanding to the eighth order:

$$
\begin{aligned}
\log x_{t+1}= & \log x+\lambda-\lambda x=\log x+\lambda-\lambda e^{\log x}, \\
\log x_{t+1}= & -2 \log x-\frac{3}{2 !}(\log x)^{2}-\frac{3}{3 !}(\log x)^{3} \\
& -\cdots-\frac{3}{8 !}(\log x)^{8} .
\end{aligned}
$$

The coefficient of each polynomial term is then truncated at the fourth decimal place:

$$
\begin{aligned}
\log x_{t+1}= & -2 \log x-1.5(\log x)^{2}-0.5(\log x)^{3}-\cdots \\
& -0.0006(\log x)^{7}-0.0001(\log x)^{8}
\end{aligned}
$$

Model III, $G_{3}(x)$, is obtained by expanding the righthand side of Eq. (4) in a Fourier series over the range $0 \leq \tilde{x} \leq \pi$. This series is then truncated at the 10 th order to yield

$$
x_{t+1}=\frac{a_{0}}{2}+\sum_{i=1}^{10}\left[a_{i} \cos \left(2 i x_{t}\right)+b_{i} \sin \left(2 i x_{t}\right)\right],
$$

where the coefficients $a_{i}$ and $b_{i}$ are obtained by

$$
\begin{aligned}
& a_{i}=\frac{2}{\pi} \int_{0}^{\pi} x e^{\lambda(1-x)} \cos (2 i x) d x \quad \text { and } \\
& b_{i}=\frac{2}{\pi} \int_{0}^{\pi} x e^{\lambda(1-x)} \sin (2 i x) d x .
\end{aligned}
$$

Model IV, $G_{4}(x)$, is obtained by expanding the righthand side of Eq. (4) by Laguerre polynomials truncated at the 20th term:

$$
x_{t+1}=\sum_{i=0}^{20} c_{i} L_{i}(x)
$$

where

$$
L_{i}(x)=\sum_{k=0}^{i}\left[(-1)^{k} / k !\right]\left(\begin{array}{l}
N \\
k
\end{array}\right) x^{k}
$$

are the Laguerre polynomials and the coefficients $c_{i}$ are obtained by 

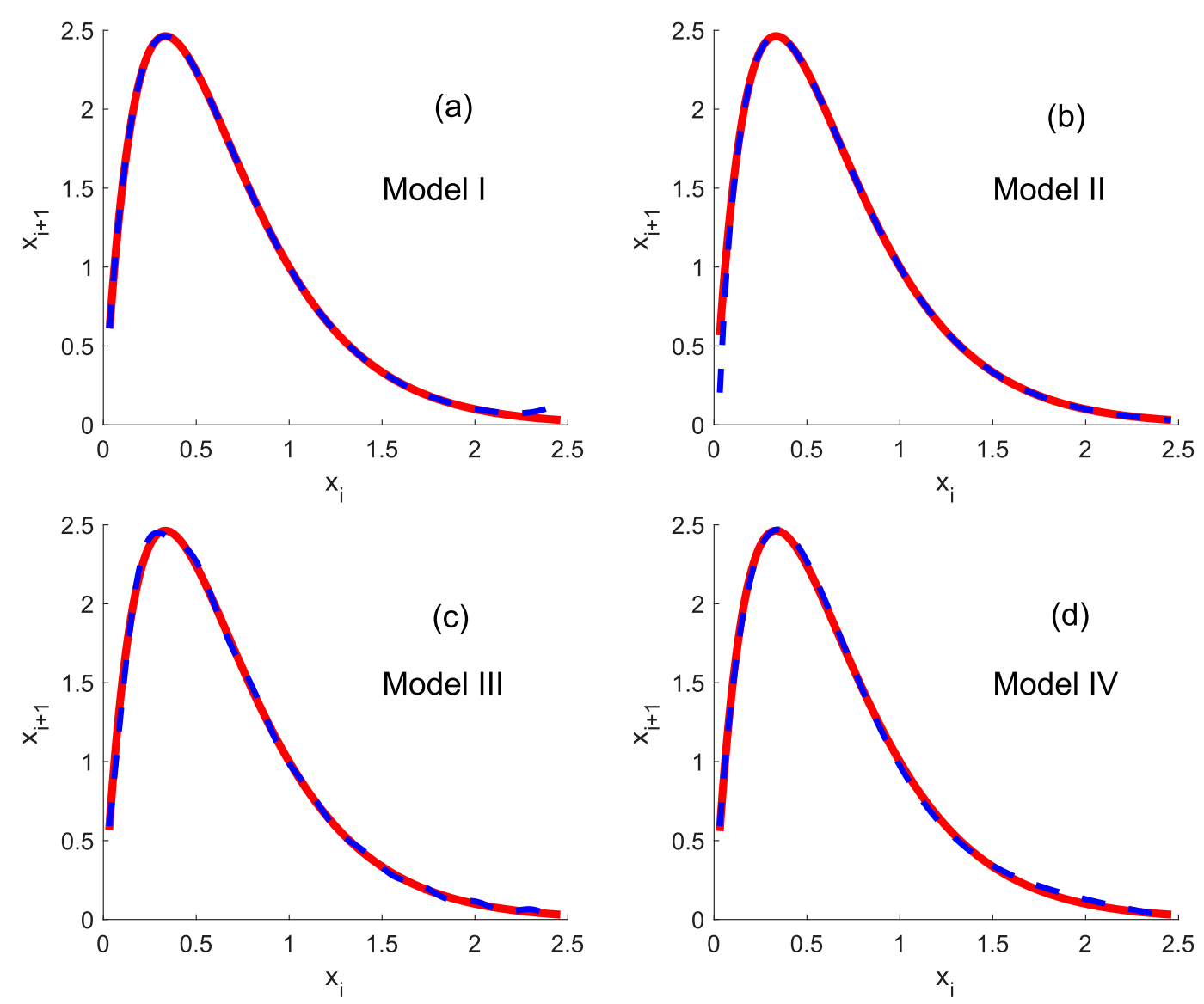

FIG. 2. Graphical presentation of the dynamics of four different models; the blue line represents model dynamics as a function of initial conditions, and the red line represents the system dynamics.

$$
c_{i}=\int_{0}^{\infty} w(x) L_{i}(x) x e^{\lambda(1-x)} d x
$$

with the weighting function $w(x)=e^{-x}$. Laguerre polynomials are orthogonal and orthonormal.

Notice that the order of the truncation for models I, II, III, and IV differ. These are chosen so that each model represents the system dynamics well and the scales of their forecast skill are comparable. Figure 2 plots the dynamical function of each model together with the system dynamics. Figure 3 presents the histogram of the one-step model error over 2048 different initial conditions that are uniformly sampled between the minimum and maximum of the Moran-Ricker system. It appears that model I simulates the system dynamics well except when the initial condition is near the maximum value of the system. For model II, a large difference between the model dynamics and the system dynamics appears only when the initial condition is near the minimum value obtained by the system. Model III does not match the system dynamics well where $x \geq 1.5$ and where the forward model reaches the maximum value of the map. Model IV matches the system less well for initial conditions near the maximum value of the map.

Figure 4 plots the two-step model error for each model, and Fig. 5 presents the histogram of the two-step model error. In general, the structure of the model error is different. Different models have different scales of model error in different local state space.

Again, there is, of course, no suggestion that the Moran-Ricker system resembles the dynamics of Earth. Rather, the framework presented here [and in (Higgins (2015)] provides probabilistic forecasts from structurally flawed models; the model-based forecasts (and ideal probabilistic forecasts formed using the perfect model) differ nontrivially from each other, and as the models are nonlinear the forecast distributions are nonGaussian. It is these challenges to multimodel forecast system development that are illustrated in this paper, which should (of course) not be taken to present an actual geophysical forecast system; indeed the verifications in the observational record rules out examination of LAP in geophysical systems, while computational 

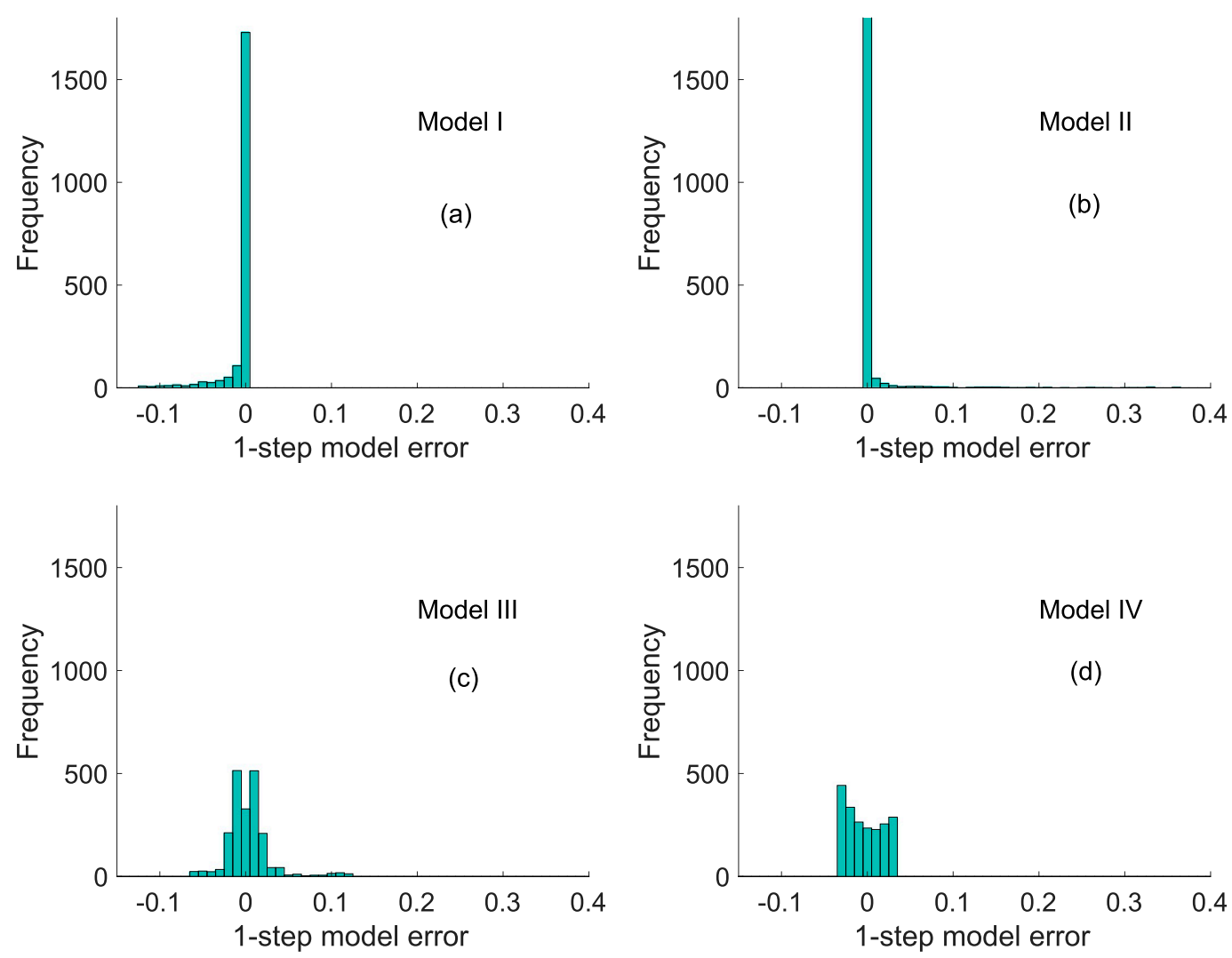

FIG. 3. Histogram of the one-step model errors, given 2048 different initial conditions with respect to natural measure.

requirements rule out extensive examination of SAP in "state of the art" geophysical models.

\section{Ensemble formation}

\section{a. Initial condition ensembles for each model}

In the experiments presented in this paper, each model produces ensemble forecasts by iterating an ensemble of initial conditions (IC). The initial condition ensemble is formed by perturbing the observation with random draws from a Normal distribution, $N\left(0, \kappa_{\tau}^{2}\right)$. If the model were perfect and the observation were exact, $\kappa_{\tau}$ would be zero; because neither of these conditions is met one does not expect $\kappa_{\tau}$ to be zero. Such a perturbation parameter $\kappa_{\tau}$ is chosen to minimize the ignorance score at lead time $\tau$. When making medium-range forecasts, the European Centre for Medium-Range Weather Forecasts (ECMWF) selects a perturbation size such that the RMS error between the ensemble members and the ensemble mean at a lead time of two days is roughly equal to the RMS of the ensemble mean and the outcome at two days.

In the experiments presented below, each initial condition ensemble will contain $N_{e}=9$ members, following the ENSEMBLES protocol. Consider first the case of a large archive, with $N_{a}=2048$. For a given $\kappa$ and lead time $\tau$, the kernel dressing and climatology-blend parameter values are fitted using a training forecast-outcome archive that contains $N_{l}=2048$ forecast-outcome pairs. The ignorance score is then calculated using an independent testing forecast-outcome set that contains $N_{t}=2048$ forecast-outcome pairs. Figure 6a shows the optimal perturbation parameter $\kappa$ for each model varies with lead time. ${ }^{5}$ The ignorance score for each model at different lead time, using the values of $\kappa$ in Fig. 6a, is shown in Fig. 6b. The sampling uncertainty across forecast launches is represented by a bootstrap resampling procedure, which resamples the set of forecast ignorance scores for each model, with replacement. The bootstrap resampling intervals are shown as vertical bars in Fig. 6 as a 5\%-95\% interval. As seen in Fig. 6a, for each model, the preferred value of $\kappa$ varies significantly

\footnotetext{
${ }^{5}$ As noted by a reviewer, there is uncertainty in the $\kappa$ values reported in Fig. 6a. To quantify this uncertainty, the estimate of $\kappa$ was bootstrap resampled. The results (not shown) show variation in $\kappa$, at lead time 1 being always less than $50 \%$, but very little variation in the corresponding ignorance value for each model.
} 

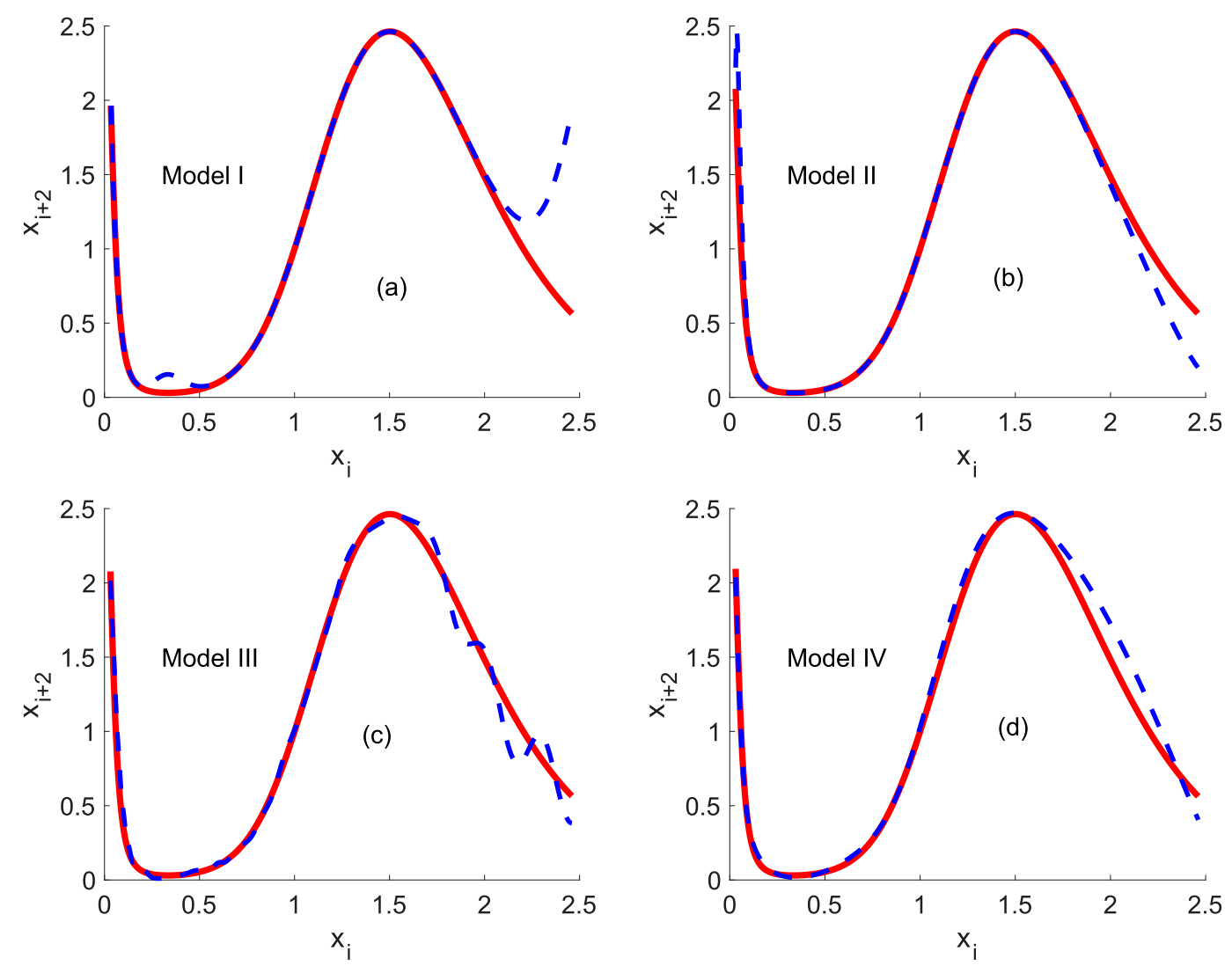

FIG. 4. Graphical presentation of the two-step evolution of four different models; the blue line represents the two-step model evolution as a function of initial conditions, and the red line represents the two-step evolution under the system.

(by about a factor of 2) between different forecast lead times. Defining a $N_{e}$-member forecast system requires selecting a specific value of $\kappa$ for each model. In this paper, the value of $\kappa$ for each model is chosen by optimizing the forecast ignorance score at lead time 1. Sensitivity tests have been conducted and the ignorance score at other lead times is much less sensitive to $\kappa$ than that at lead time 1. Bias correction in the dressing blending approach is another concern. Hodyss et al. (2016) discussed bias in a real-world context. The dressing blending approach can be generalized by including a shifting parameter (see Bröcker and Smith 2008) to account for model bias. Including the shifting parameter does, in fact, improve the ignorance score out-of-sample (in each model at almost all lead times) in this case. As the improvement is typically less than one 20 th of a bit (sometimes zero), such shifting parameter is not included in the dressing blending throughout the experiments presented in the current paper.

\section{b. On the number of IC simulations in each ensemble}

Forecast system design relies on the knowledge of the relationship between the size of the forecast ensemble and the information content of the forecast (Smith et al. 2015). Usually, the cost of developing a brand new model is tremendously larger than the cost of increasing the number of ensemble members. ${ }^{6}$ Furthermore, the cost of increasing the ensemble size increases only (nearly) linearly and decreases as technology improves.

As the number of ensemble members increases, the true limitation due to structural model error becomes more apparent. Figure 7 shows that forecast ignorance varies as ensemble size increases. Improvement from the additional ensemble members can be noted, especially at shorter lead times.

\section{Forecast system design and model weighting when data are precious}

\section{a. Forecasts with a large forecast-outcome archive}

As $N_{a}$, the size of the forecast-outcome archive, increases, one expects robust results since large training

\footnotetext{
${ }^{6}$ In financial terms, the cost falls on the current account and not on the capital account.
} 

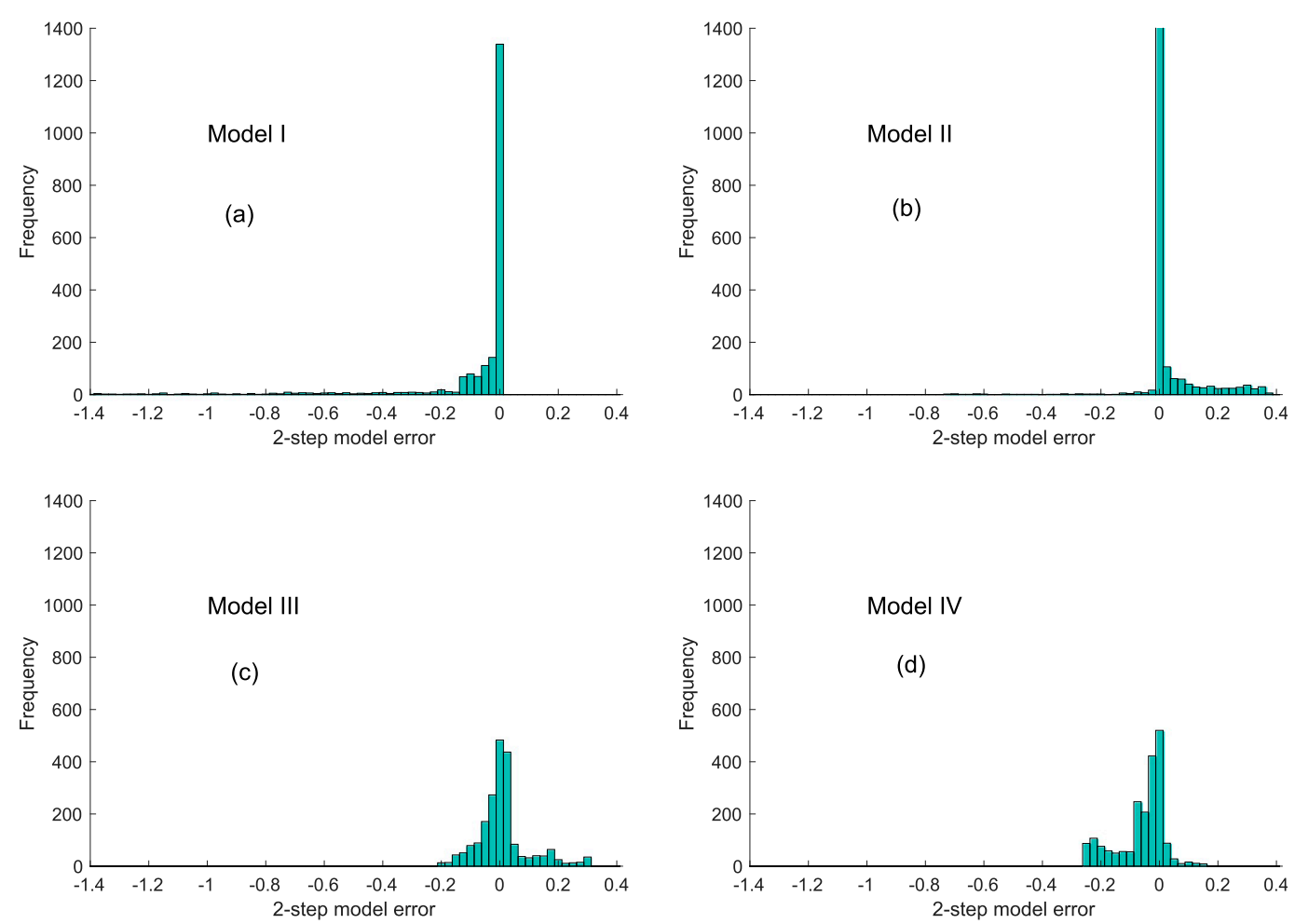

FIG. 5. As in Fig. 3, but for the two-step model.

sets and large testing sets are considered. To examine this, 512 different training sets are produced, each contains 2048 forecast-outcome pairs. For each archive, the kernel width $\sigma$ and climatology-blend weight $\alpha$ are fitted for each model's forecasts at lead time. Figures $8 \mathrm{a}$ and $8 \mathrm{~b}$ show the fitted values of the dressing parameters and climatology-blend weights. The error bars reflect the central 90th percentile over 512 samples. The variation of the weight assigned to the model appears small. The variation of the fitted kernel width is small at short lead times and large at long lead times. Especially at lead time 5, the fitted value for Model IV has relatively large
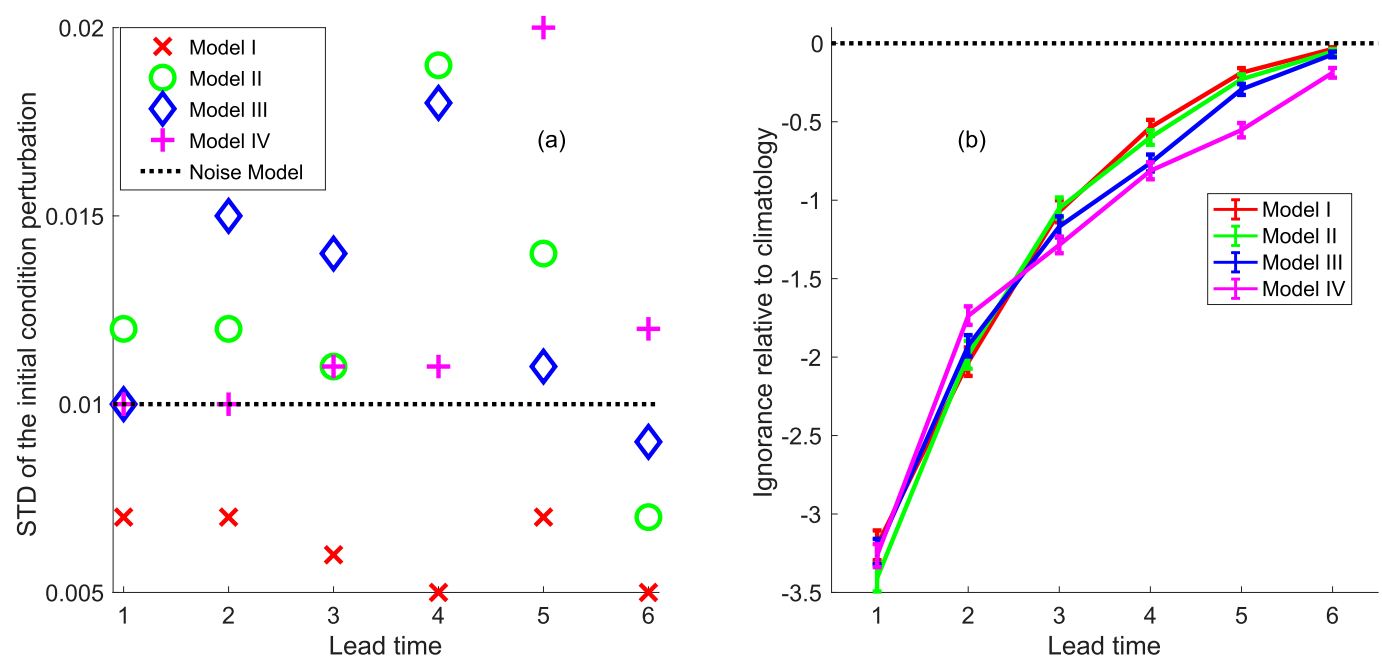

FIG. 6. (a) The best found perturbation parameter values $\kappa$ as a function of lead time for each model; the dashed black line reflects the standard deviation of the noise model. (b) Ignorance score of each model as a function of lead time; the dashed black line reflects skill of climatology, which defines zero skill. 

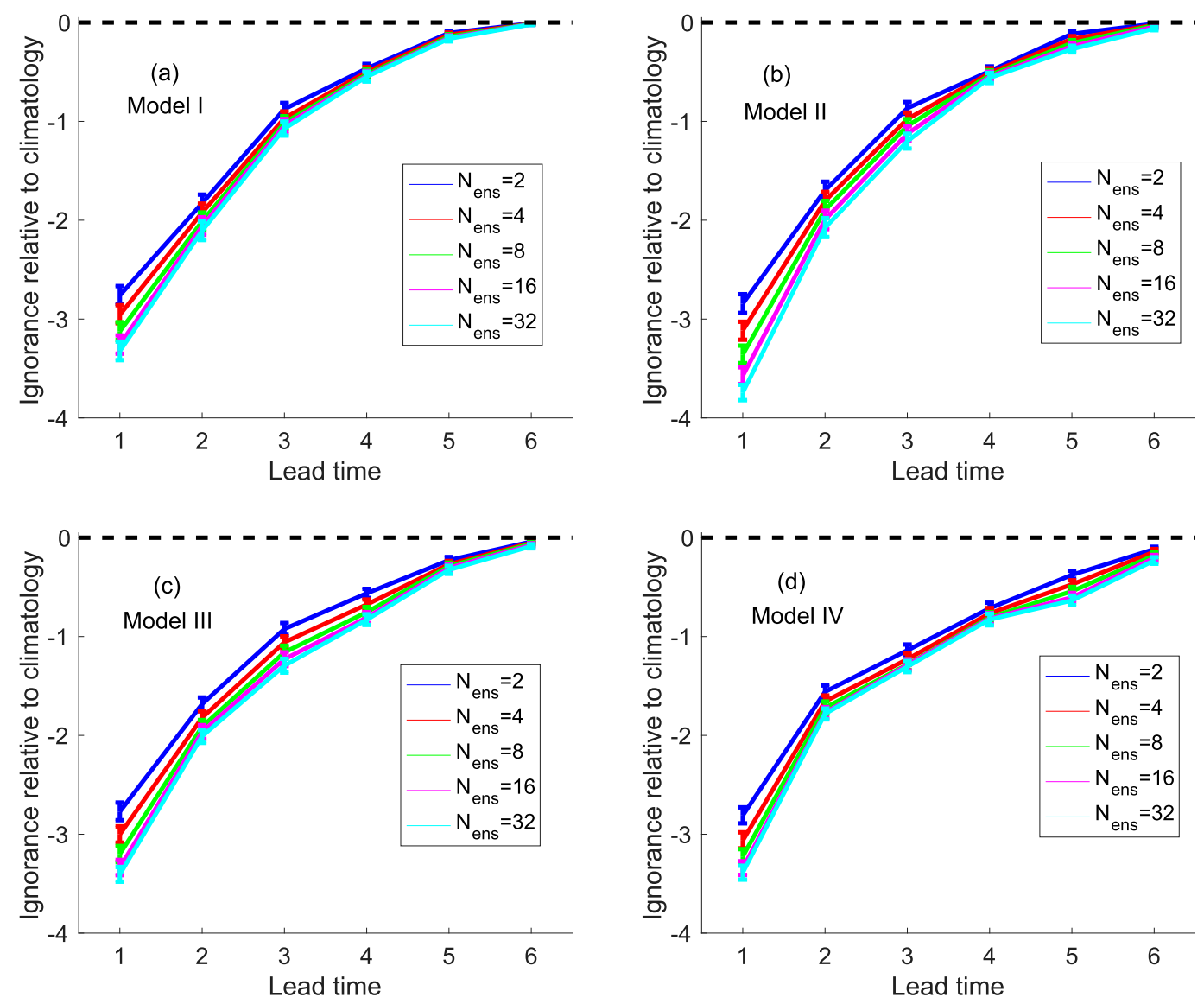

FIG. 7. The ignorance score; it varies as the ensemble size increases for each model.

variation. This, however, does not indicate that the estimate is not robust but suggests the ignorance score function in the parameter space is relatively flat near the minimum. To demonstrate this, the empirical ignorance is calculated for each archive of kernel width and climatology-blend weight based on the same testing set (which contains another 2048 forecast-outcome pairs). Figure $8 \mathrm{c}$ plots the ignorance score and its 90th percentile as a function of lead time. Notice that the 90th-percentile ranges are always very narrow.

The next two paragraphs echo Smith et al. (2015). There are many ways to combine multiple single model forecast distributions into a single probabilistic (multimodel) forecast distribution (Hagedorn et al. 2005; Bröcker and Smith 2008). A simple approach is to treat each model equally and therefore apply equal weight to each individual model (see e.g., Weisheimer et al. 2009). In general, different models perform differently in terms of forecasts, for example, the ECMWF model significantly outperforms other models in seasonal forecasts (Smith et al. 2015). Therefore, applying nonequal weights to all contributing models might provide more skillful multimodel forecast distribution (see e.g., Rajagopalan et al. 2002). Following DoblasReyes et al. (2005) and Smith et al. (2015), define the combined multimodel forecast distribution to be the weighted linear sum of the constituent distributions:

$$
p_{\mathrm{mm}}=\sum_{i} \omega_{i} p_{i}
$$

where $p_{i}$ is the individual forecast distribution from the $i$ th model and $\omega_{i}\left(\sum_{i} \omega_{i}=1\right)$ is the corresponding weight. The weighting parameters $\omega_{i}$ may be determined according to their performance in a past forecast-outcome archive. The weights of individual models are expected to vary as a function of lead time.

It is computationally costly and potentially results in ill-fitted model weights if all of the weights are fitted simultaneously. To avoid both issues, a simple iterative approach (Du and Smith 2017) is adopted. For each lead time, the best (in terms of ignorance) model is first combined with the second-best model to form a combined forecast distribution (by assigning weights to both models that optimize the ignorance of the combined 

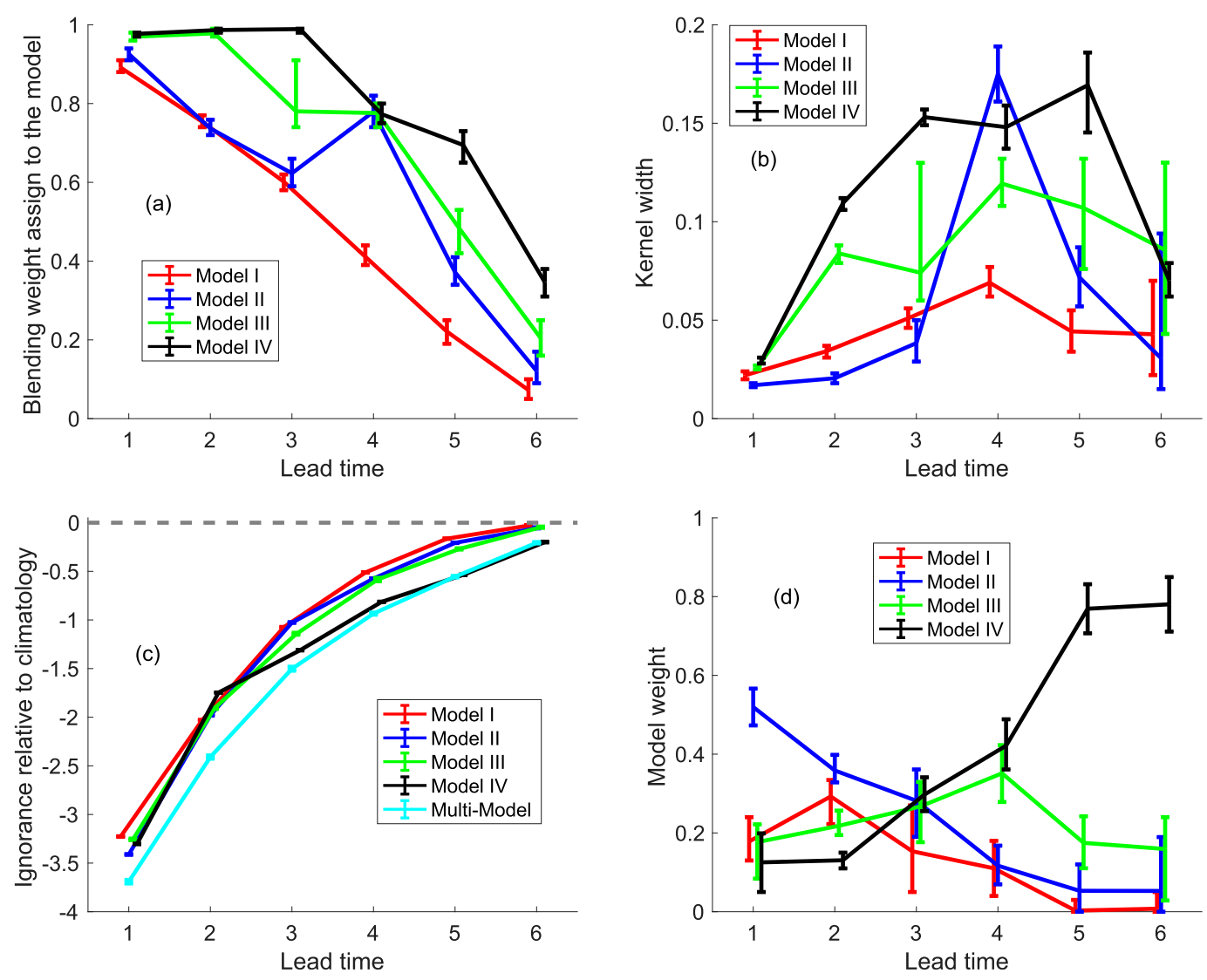

FIG. 8. (a) Climatology-blend weight assigned to the model, (b) kernel width, (c) forecast ignorance, and (d) weights assigned to each individual model are plotted as a function of lead time.

forecast). The combined forecast distribution is then combined with the third-best model to update the combined forecast distribution. This process is repeated until inclusion of the "worst" model is considered. Note each time a new model is included in the combined model, only two weights need to be assigned. Figure $8 \mathrm{~d}$ shows the weights assigned to each model as a function of lead time. The cyan line in Fig. $8 \mathrm{c}$ shows the variation of the ignorance score for the multimodel forecast given those estimated model weights is very small.

\section{b. Forecast with a small forecast-outcome archive}

When given a small forecast-outcome archive (e.g., from an $\sim 40$-yr seasonal forecast-outcome archive), one does not have the luxury of exploring a large collection of independent training and testing sets. Cross validation is often approached by adopting a leave-one-out approach. The robustness of parameter fitting in such cases is of concern. To examine such robustness, a large number of forecast-outcome archives are considered. Each archive contains the same numbers of forecast-outcome pairs. For each archive, the parameter values are fitted via leave-one-out cross validation. The distribution of fitted values over these small forecast-outcome archives is then compared with the fitted value from the $N_{a}=2048$ forecast-outcome archives above. Figure 9 plots the histograms of the fitted climatology-blend weights given 512 forecastoutcome archives each containing $N_{a}=40$ forecastoutcome pairs. Notice that, in most cases, the distributions are very wide although they cover the value fitted given the large training set. There are some cases in which about $90 \%$ of the estimates are larger or smaller than the values fitted by the large archive (e.g., lead time 1 of model I and model II and lead time 4 of model III and model IV). It therefore appears that the robustness of fitting varies with lead time and the model. For shorter lead times, however, the weights are more likely to be overfitted and, for longer lead times, the weights are more likely to be underfitted. This is because at short lead times the model forecasts are relatively good; only a few forecast systems yield predictions that are worse than the climatological forecast. Small forecastoutcome archives, on the other hand, may not contain any model busts and so often overestimate the weights. 

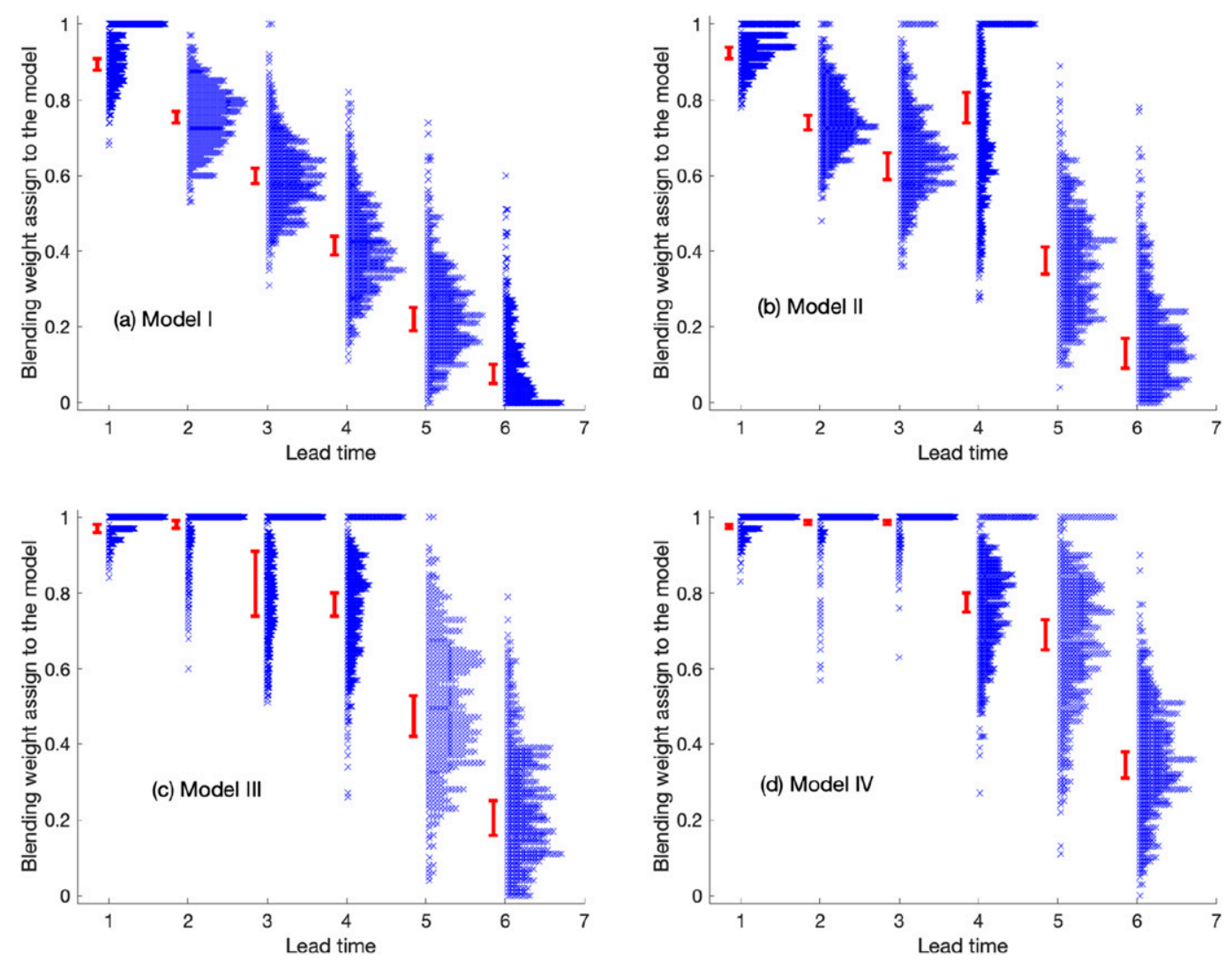

FIG. 9. Climatology-blend weights assigned to each model. The red bars are the 95th percentile range of the fitted weights based on 512 forecast-outcome archives. Each contains 2048 forecast-outcome pairs. The blue crosses represent the histogram of the fitted weights based on 512 forecast-outcome archives. Each of these contains only 40 forecast-outcome pairs.

The longer lead time case can be explained similarly. Figure 10 plots the histogram of fitted kernel widths. Again, observe that there is much larger variation of the estimates here than when fitting with large forecastoutcome archives.

Poor estimation of the kernel width and climatologyblend weight will cause the forecast to lose skill and appear to underperform out-of-sample (due to inappropriately high expectations). This could, of course, be misinterpreted as climate change. For each of the 512 fitted kernel widths and climatology-blend weights, the ignorance scores are calculated over the same testing set of 2048 forecast-outcome pairs. Figure 11 plots the histogram of the ignorance score for each model. Using parameters fitted with small archives often results in significant degrading $(\sim 1 \mathrm{bit})$ of the ignorance score of the forecasts. Correctly blending with the climatological distribution would yield a forecast score that, in expectation, is never worse than the climatology. When the blending parameter is determined using the small archive, however, the average relative ignorance can be worse than climatology out-of-sample at long lead times (see e.g., in Fig. 11). Figure 12 plots the histogram of multimodel weights. Clearly the variation of the model weights based on a small archive are much larger. Weights of zero are often assigned to model forecasts that contain useful information, for example.

\section{Multimodel versus single best model}

As noted in Smith et al. (2015), ${ }^{7}$ it is sometimes said that a multimodel ensemble forecast is more skillful than any of its constituent single-model ensemble forecasts (see e.g., Palmer et al. 2004; Hagedorn et al. 2005; Bowler et al. 2008; Weigel et al. 2008; Weisheimer et al. 2009; Alessandri et al.2011). One common "explanation" (Weigel et al. 2008; Weisheimer et al. 2009; Alessandri et al. 2011) for this is that individual model tends to be overconfident with its forecast and a multimodel forecast reduces such overconfidence, which leads

\footnotetext{
${ }^{7}$ These first two sentences are taken from Smith et al. (2015).
} 

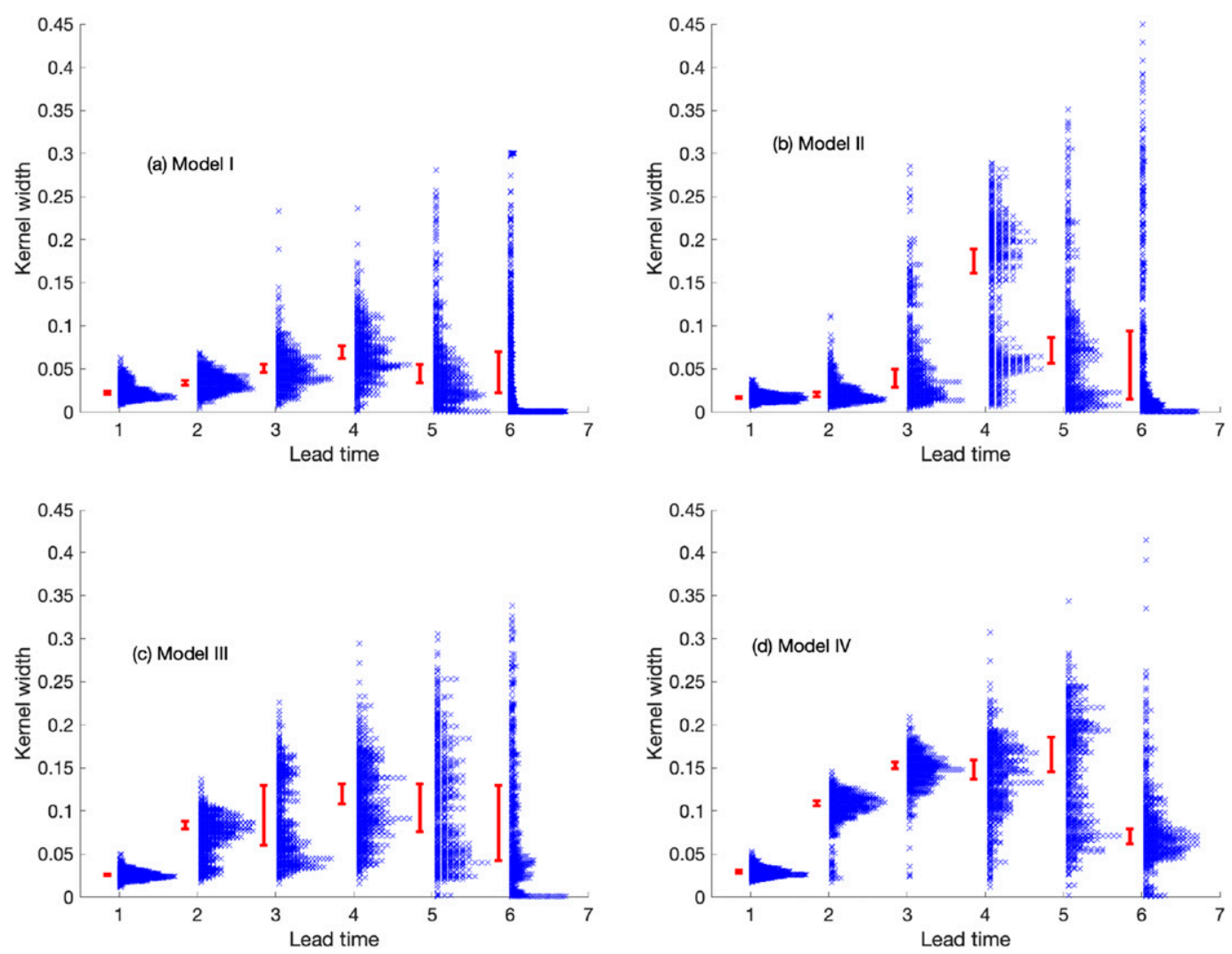

FIG. 10. Kernel width of each model's forecasts. The red bars are the 95th percentile range of the fitted kernel width based on 512 forecast-outcome archives. Each contains 2048 forecast-outcome pairs. The blue crosses represent the histogram of the fitted kernel width based on 512 forecast-outcome archives. Each of these contains only 40 forecast-outcome pairs.

to a more skillful forecast performance. As shown in section 6 , single model SAP forecast systems are typically between half a bit and two bits less skillful than an LAP system based on the same model. Can a SAP multimodel forecast system regain some of this potential skill? Fig. 12 shows that this is unlikely, as the determination of model weights given SAP varies tremendously relative to their LAP values. Again, it is the performance of the combination of weights that determine the skill of the forecasts, so this variation need not always be deadly.

Figure 13 shows the skill of the multimodel forecast system relative to the forecast system based on the single best model. Both the SAP and the LAP forecast systems show that the multimodel system usually outperforms the single model. Comparing SAP multimodel systems with the single best model SAP system (Fig. 13b), the advantage of the multimodel system(s) is stronger when the best model (as well as all the parameters: model weights and dressing and climatologyblended parameters) are ill identified. Comparing SAP multimodel systems with the single best model LAP system (Fig. 13c), however, the advantage of the multimodel system(s) is weaker. Multimodel systems do not always outperform the single best model, especially at longer lead times.

At this point, one faces questions of resource distribution. A fair comparison of an $N$-model forecast system would be against a single model with $n$-timeslarger ensemble. (This, of course, ignores the operational fact that it is much more demanding to maintain an ensemble of models than to maintain a large ensemble under one model.) Second, note that for each model, $\kappa$ was a function of lead time. At the cost of making ensemble members nonexchangeable, one could draw ensembles from distinct groups, and weight these members differently for each lead time. One also could develop methods that treat the raw ensemble members from each of the models as nonexchangeable and use a more complex interpretation to form the forecast. While the simple forecast framework of this paper is an ideal place to explore such questions, they lie beyond the scope of this paper. Instead, the extent to which the multimodel forecast system is more 

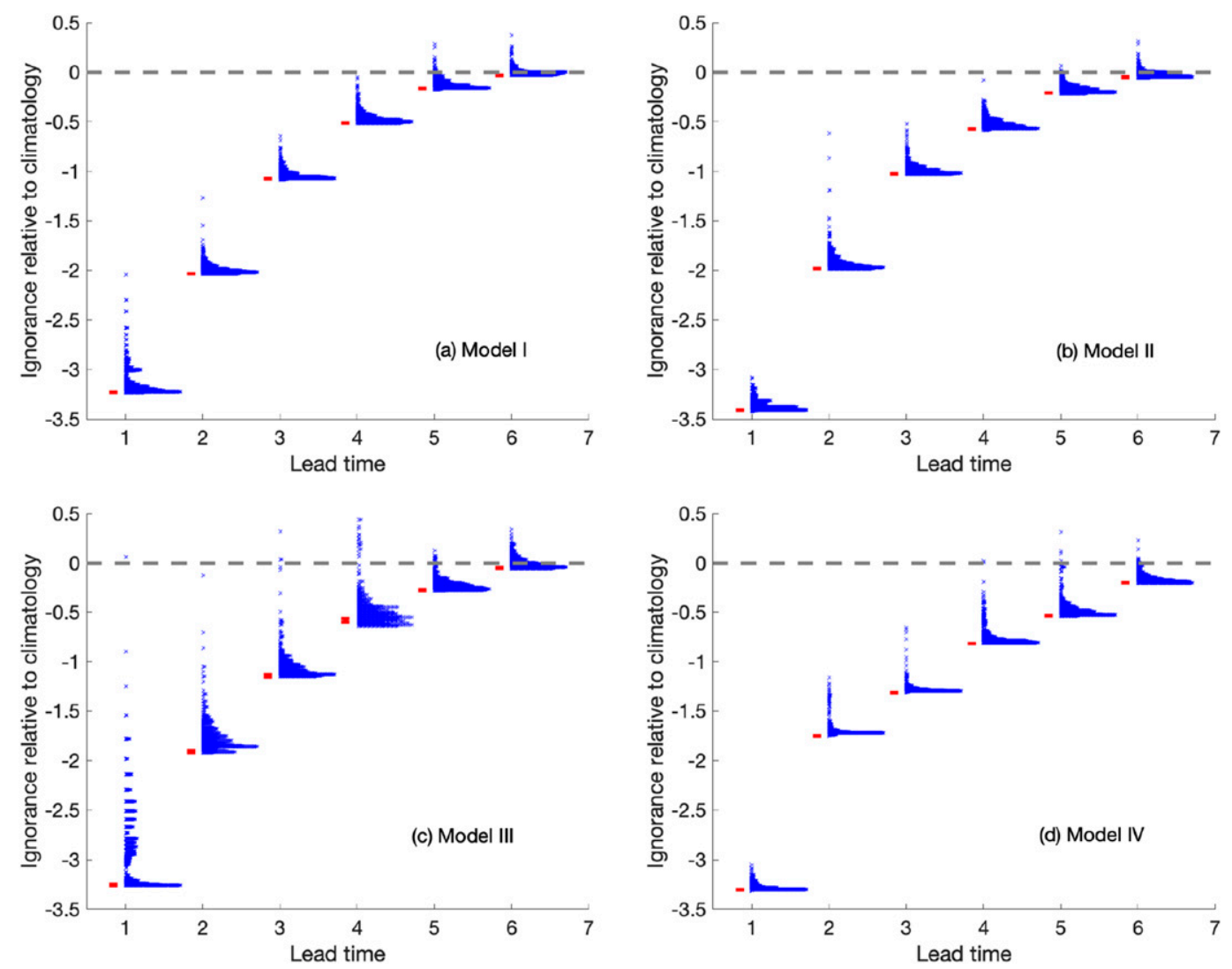

FIG. 11. Ignorance score of each model's forecasts. The red bars are the 95th percentile range of ignorance score calculated based on a testing set containing 2048 forecast-outcome pairs, using the climatology-blend weights and kernel widths fitted based on 512 forecast-outcome archives. Each contains 2048 forecast-outcome pairs. The blue crosses represent the histogram of ignorance score calculated based on the same testing set but using the climatology-blend weights and kernel widths based on 512 forecast-outcome archives. Each contains only 40 forecast-outcome pairs.

misleading than the single model systems concludes the discussion in the next section.

\section{Discussion and conclusions}

A significant challenge to the design of seasonal probabilistic forecasting has been discussed and illustrated in a simple system where multiple models can easily be explored in long time limits. The challenge has been addressed within the surrogate modeling paradigm. In the actual system of interest, empirical data are precious: we have very few relevant out-of-sample forecasts, and doubling the current sample size will take decades. For these reasons we consider surrogate systems with sufficient similarity given the questions we wish to ask. We are forced to assume that the results obtained are general enough to make them informative for design in the real-world system; in this particular case we believe that they are: the challenges of interpreting small ensembles in any multimodel context are arguably very similar. Similarly, the convergence to a clear conclusion in the limit of large ensembles is also arguably quite similar. The details of the rate at which information increases as the ensemble size increases will depend on the details of the dynamics of the system, the quality of the models, and so on. That said, there is sufficient evidence from the study above to show that some current multimodel ensemble studies do not employ initial condition ensembles of sufficient size to achieve robust results.

There is no statistical fix to the challenges of "lucky strikes" when a generally poor model places an ensemble member near an outcome "by chance", and that particular outcome was not well predicted by the other forecast systems. Similarly "hard busts" in a small archive can distort the parameters of the forecast systems, when an outcome occurs relatively far from each ensemble member. In this case, wider kernels and/or heavier weighting on the climatology results. This may be due to structural model failure or merely to a "rare" event, where rare is related to the ensemble size. Given a 

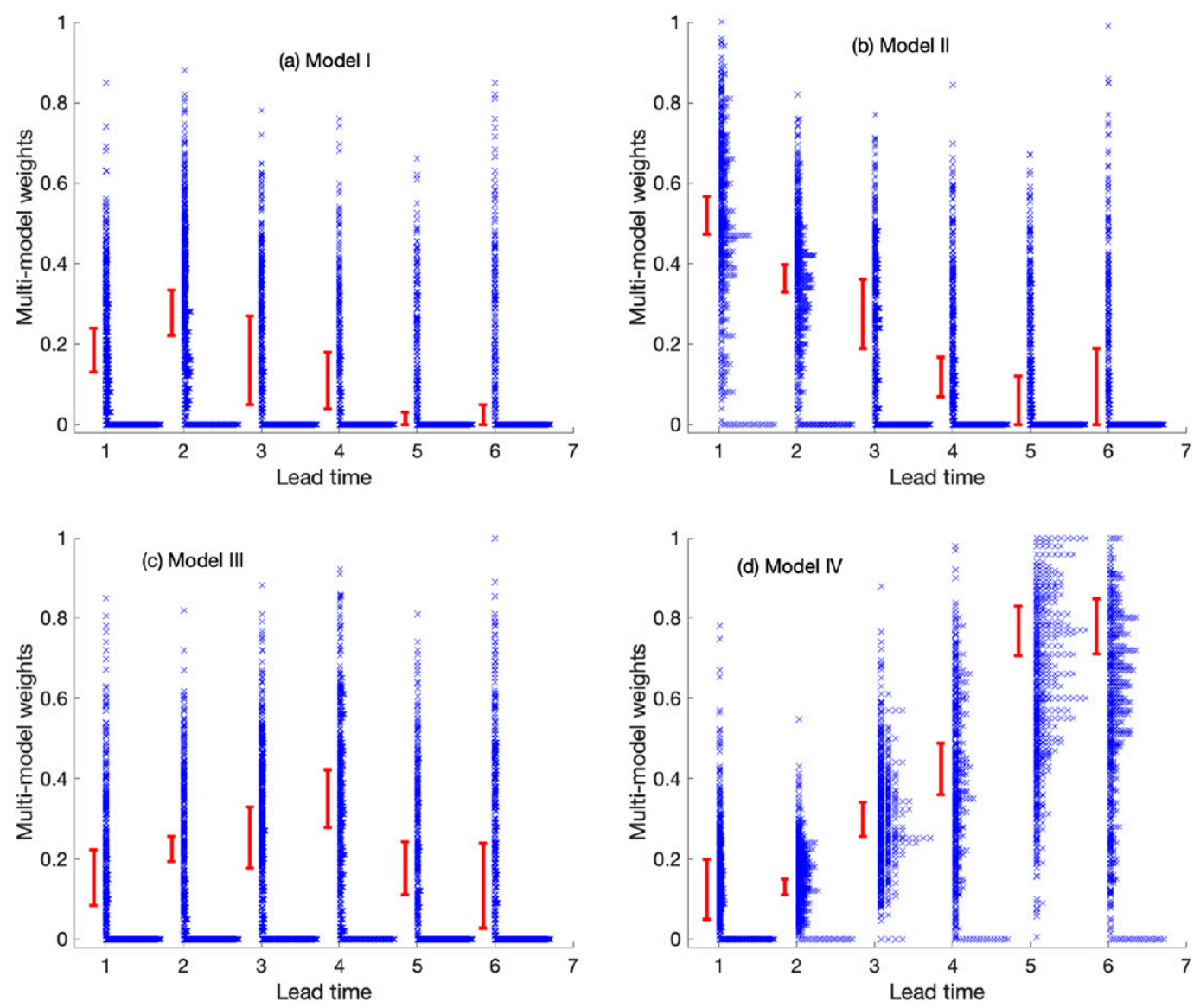

FIG. 12. Multimodel weights for each set of model forecasts. The red bars are the 95th percentile range of model weights calculated based on a testing set containing 2048 forecast-outcome pairs, using the climatology-blend weights and kernel widths fitted based on 512 forecast-outcome archives. Each contains 2048 forecast-outcome pairs. The blue crosses represent the histogram of model weights calculated based on the same testing set but using the climatology-blend weights and kernel widths based on 512 forecast-outcome archives. Each contains only 40 forecast-outcome pairs.

sufficiently large ensemble, the forecast system could have assigned an (appropriately low) probability to the observed "bust" event.

In short, the brief duration of the forecast-outcome archive, typically less than 40 years in seasonal forecasting, limits the clarity both with which probability distributions can be derived from individual models and with which model weights can be determined. No clear solution to this challenge has been proposed, and while improvements on current practice can be made, it is not clear that this challenge can be met. Over long periods, like 512 years, the climate may not be well approximated as stationary. In any event, both observational systems and the models themselves can evolve significantly on much shorter time scales, perhaps beyond recognition.

One avenue open to progress is in determining the relative skill of "the best model" (or a small subset) and the full diversity of models. Following Bröcker and
Smith (2008) it is argued that a forecast system under the best model with a large ensemble may well outperform the multimodel ensemble forecast system when both systems are given the same computer power. To test this in practice requires access to larger ensembles under the best model. This paper argues future studies, such as ENSEMBLES, could profitably adjust their experimental design to take this into account (see also Machete and Smith 2016).

A second avenue is to reduce the statistical uncertainty of model fidelity within the available archive. This can be done by running large ensembles (much greater than " 9 ", indeed greater than might be operationally feasible) under each model. This would allow identification of which models have significantly different probability distributions, and the extent to which they are (sometimes) complementary. Tests with large ensembles also reveal the "bad busts" that are due to 

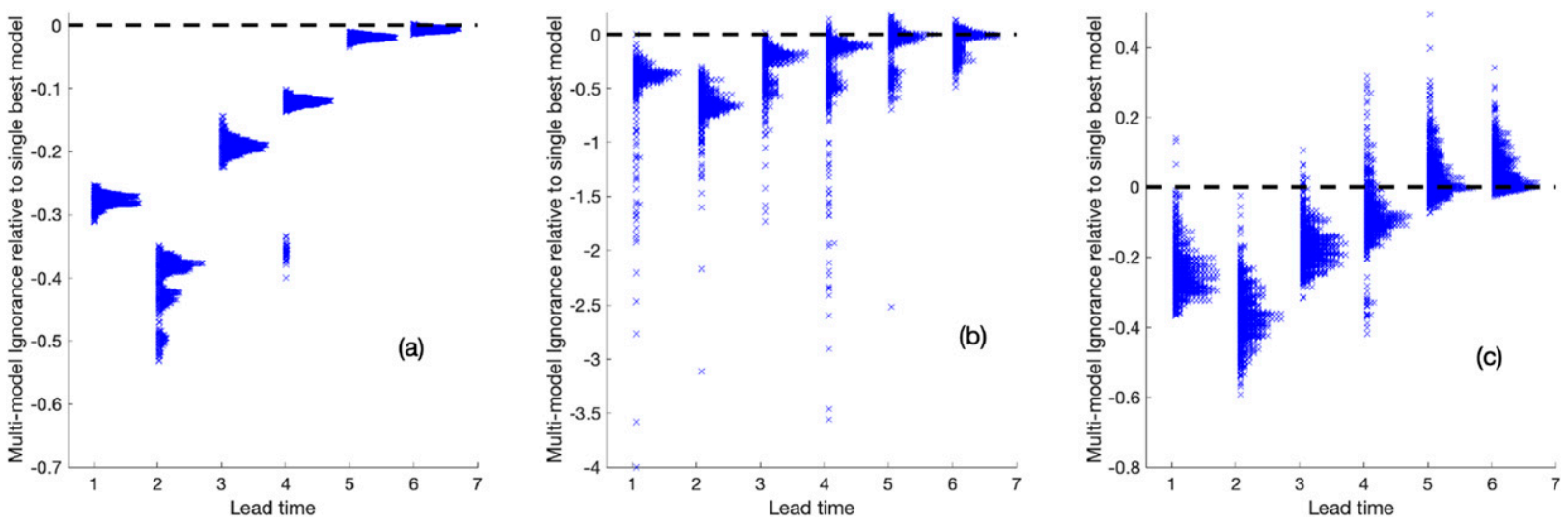

FIG. 13. Ignorance of multimodel ensemble relative to the single best model. The blue crosses represent the histogram of the ignorance of the multimodel ensemble relative to the single best model (black dashed line). (a) Model weights and dressing and climatology-blend parameters are fitted based on 512 large archives. Each contains 2048 forecast-outcome pairs. (b) Model weights and dressing and climatology-blend parameters are fitted based on 512 small archives. Each contains 40 forecast-outcome pairs. (c) The ignorance of the multimodel ensemble is calculated using model weights and dressing and climatology-blend parameter that are fitted based on 512 small archives, whereas the ignorance of the single best model is calculated based on 512 large archives.

small ensemble size to be what they are. It can also suggest that those that remain are indeed due to structural model error.

In closing, it is suggested that perhaps the most promising way forward is to step away from the statistics of the ensembles and to consider the physical realism of the individual trajectories. One can look for shadowing trajectories in each model and attempt to see what phenomena limit the model's ability to shadow. Identifying these phenomena, and the phenomena that cause them, would allow model improvement independent of the probabilistic skill of ensemble systems. This approach is not new, of course, but is the traditional physical approach to model improvement that dates back to Charney. Modern forecasting methods do offer some new tools (Judd et al. 2008), and the focus on probabilistic forecasting is well placed in terms of prediction. The point here is merely that probabilistic forecast skill, while a sharp tool for decision support, may prove a blunt tool for model improvement when the data are precious.

Acknowledgments. This research was supported by the LSE's Grantham Research Institute on Climate Change and the Environment and the ESRC Centre for Climate Change Economics and Policy, funded by the Economic and Social Research Council and Munich Re; it was also supported as part of the EPSRC-funded Blue Green Cities (EP/K013661/1). Additional support for author Du was also provided by the EPSRC-funded uncertainty analysis of hierarchical energy systems models: models versus real energy systems (EP/K03832X/1) and Centre for Energy Systems Integration (EP/P001173/1).
Author Smith gratefully acknowledges the continuing support of Pembroke College, Oxford.

\section{APPENDIX}

\section{From Simulation to a Predictive Distribution}

This appendix is taken from Smith et al. (2015) appendix A.

An ensemble of simulations is transformed into a probabilistic distribution function by a combination of kernel dressing and blending with climatology (Bröcker and Smith 2008). An $N$-member ensemble at time $t$ is given as $X_{t}=\left(x_{t}^{1}, \ldots, x_{t}^{N}\right)$, where $x_{t}^{i}$ is the value of an observable quantity for the $i$ th ensemble member. For simplicity, ensemble members given a model are considered to be exchangeable. Kernel dressing defines the model-based component of the density as

$$
p(y: X, \sigma)=\frac{1}{N \sigma} \sum_{i}^{N} K\left[\frac{y-\left(x^{i}\right)}{\sigma}\right],
$$

where $y$ is a random variable (the correspondent of the density function $p$ ) and $K$ is the kernel, taken here to be

$$
K(\zeta)=\frac{1}{\sqrt{2 \pi}} \exp \left(-\frac{1}{2} \zeta^{2}\right)
$$

Thus, each ensemble member contributes a Gaussian kernel centered at $x^{i}$. For a Gaussian kernel, the kernel width $\sigma$ is simply the standard deviation determined empirically as discussed below. 
Even for an ensemble drawn from the the same distribution as the outcome, there remains the chance of $\sim 2 / N$ that the outcome lies outside the range of the ensemble. Given the nonlinearity of the model, such outcomes can be very far outside the range of the ensemble members. In addition to $N$ being finite, the simulations are not drawn from the same distribution as the outcome, because the forecast system is never perfect in practice. To improve the skill of the probabilistic forecasts, the kernel dressed ensemble may be blended with an estimate of the climatological distribution of the system obtained by dressing the historical observations [see Bröcker and Smith (2008) for more details, Roulston and Smith (2003) for alternative kernels, and Raftery et al. (2005) for a Bayesian approach]. The blended forecast distribution is then written as

$$
p()=\alpha p_{m}()+(1-\alpha) p_{c}()
$$

where $p_{m}$ is the density function generated by dressing the model ensemble and $p_{c}$ is the estimate of the climatological density. The blending parameter $\alpha$ determines how much weight is placed on the model. Specifying both values (kernel width $\sigma$ and climatology blended parameter $\alpha$ ) at each lead time defines the forecast distribution. Both parameters are fitted simultaneously by optimizing the empirical ignorance score over the training set.

\section{REFERENCES}

Alessandri, A., A. Borrelli, A. Navarra, A. Arribas, M. Déqué, P. Rogel, and A. Weisheimer, 2011: Evaluation of probabilistic quality and value of the ensembles multimodel seasonal forecasts: Comparison with DEMETER. Mon. Wea. Rev., 139, 581-607, https://doi.org/10.1175/2010MWR3417.1.

Bernardo, J. M., 1979: Expected information as expected utility. Ann. Stat., 7, 686-690, https://doi.org/10.1214/aos/ 1176344689.

Bougeault, P., and Coauthors, 2010: The Thorpex Interactive Grand Global Ensemble (TIGGE). Bull. Amer. Meteor. Soc., 91, 1059-1072, https://doi.org/10.1175/2010BAMS2853.1.

Bowler, N. E., A. Arribas, and K. R. Mylne, 2008: The benefits of multianalysis and poor man's ensembles. Mon. Wea. Rev., 136, 4113-4129, https://doi.org/10.1175/2008MWR2381.1.

Bröcker, J., and L. A. Smith, 2007: Scoring probabilistic forecasts: The importance of being proper. Wea. Forecasting, 22, 382388, https://doi.org/10.1175/WAF966.1.

- , and - 2008: From ensemble forecasts to predictive distribution functions. Tellus, 60A, 663-678, https://doi.org/ 10.1111/j.1600-0870.2008.00333.x.

Doblas-Reyes, F. J., R. Hagedorn, and T. N. Palmer, 2005: The rationale behind the success of multi-model ensembles in seasonal forecasting. Part II: Calibration and combination. Tellus, 57A, 234-252, https://doi.org/10.3402/tellusa.v57I3.14658.

, A. Weisheimer, T. N. Palmer, J. M. Murphy, and D. Smith, 2010: Forecast quality assessment of the ENSEMBLES seasonal-to-decadal Stream 2 hindcasts. Tech. Memo. 621, ECMWF, 47 pp., https://www.ecmwf.int/node/9071.
Du, H., and L. A. Smith, 2017: Multi-model cross-pollination in time. Physica D, 353-354, 31-38, https://doi.org/10.1016/ j.physd.2017.06.001.

Gilmour, I., and L. A. Smith, 1997: Enlightenment in shadows. Proc. AIP Conf. on Nonlinear Dynamics and Stochastic Systems Near the Millennium, San Diego, CA, American Institute of Physics, 335-340, http://www.lse.ac.uk/CATS/Assets/PDFs/Publications/ Papers/1999-and-before/Enlightenment-in-shadows-1997.pdf.

Glendinning, P., and L. A. Smith, 2013: Lacunarity and perioddoubling. Dyn. Syst., 28, 111-121, https://doi.org/10.1080/ 14689367.2012.755496.

Good, I. J., 1952: Rational decisions. J. Roy. Stat. Soc. A, 14, 107114, https://doi.org/10.1111/J.2517-6161.1952.TB00104.X.

Hagedorn, R., F. J. Doblas-Reyes, and T. N. Palmer, 2005: The rationale behind the success of multi-model ensembles in seasonal forecasting. Part I: Basic concept. Tellus, 57A, 219233, https://doi.org/10.3402/tellusa.v57I3.14657.

Hewitt, C. D., and D. J. Griggs, 2004: Ensembles-based predictions of climate changes and their impacts. Eos, Trans. Amer. Geophys. Union, 85, 566, https://doi.org/10.1029/2004EO520005.

Hide, R., 1958: An experimental study of thermal convection in a rotating fluid. Philos. Trans. Roy. Soc., A250, 441-478, https:// doi.org/10.1098/RSTA.1958.0004.

Higgins, S. M. W., 2015: Limitations to seasonal weather prediction and crop forecasting due to nonlinearity and model inadequacy. Ph.D. thesis, The London School of Economics and Political Science, $277 \mathrm{pp}$.

Hodyss, D., E. Satterfield, J. McLay, T. M. Hamill, and M. Scheuerer, 2016: Inaccuracies with multimodel postprocessing methods involving weighted, regression-corrected forecasts. Mon. Wea. Rev., 144, 1649-1668, https://doi.org/10.1175/MWR-D-15-0204.1.

Hoeting, J. A., D. Madigan, A. E. Raftery, and C. T. Volinsky, 1999: Bayesian model averaging: A tutorial. Stat. Sci., 14, 382417, https://projecteuclid.org/euclid.ss/1009212519.

Judd, K., C. A. Reynolds, T. E. Rosmond, and L. A. Smith, 2008: The geometry of model error. J. Atmos. Sci., 65, 1749-1772, https://doi.org/10.1175/2007JAS2327.1.

Kirtman, B. P., and Coauthors, 2014: The North American Multimodel Ensemble: Phase-1 seasonal-to-interannual prediction; phase-2 toward developing intraseasonal prediction. Bull. Amer. Meteor. Soc., 95, 585-601, https://doi.org/10.1175/ BAMS-D-12-00050.1.

Lorenz, E. N., 1963: Deterministic nonperiodic flow. J. Atmos. Sci., 20, 130-141, https://doi.org/10.1175/1520-0469(1963) $020<0130$ :DNF>2.0.CO;2.

__ 1995: Predictability: A problem partly solved. Seminar on Predictability, Shinfield Park, Reading, ECMWF, 1-18, https://www.ecmwf.int/node/10829.

Machete, R. L., 2007: Modelling a Moore-Spiegel electronic circuit: The imperfect model scenario. Ph.D. thesis, University of Oxford, 185 pp., https://ora.ox.ac.uk/objects/uuid:0186999b-3e624e18-9ca9-9603be0acae2/download_file?file_format=pdf\&safe filename $=$ machete.pdf\&type_of_work $=$ Thesis.

— ensembles in forecasting physical systems. Tellus, $\mathbf{6 8 A}, 28393$, https://doi.org/10.3402/tellusa.v68.28393.

Moran, P. A. P., 1950: Some remarks on animal population dynamics. Biometrics, 6, 250-258, https://doi.org/10.2307/ 3001822 .

Palmer, T. N., and Coauthors, 2004: Development of a European Multimodel Ensemble System for Seasonal-to-Interannual Prediction (DEMETER). Bull. Amer. Meteor. Soc., 85, 853872, https://doi.org/10.1175/BAMS-85-6-853. 
Raftery, A. E., T. Gneiting, F. Balabdaoui, and M. Polakowski, 2005: Using Bayesian model averaging to calibrate forecast ensembles. Mon. Wea. Rev., 133, 1155-1174, https://doi.org/ 10.1175/MWR2906.1.

Rajagopalan, B., U. Lall, and S. E. Zebiak, 2002: Categorical climate forecasts through regularization and optimal combination of multiple gcm ensembles. Mon. Wea. Rev., 130, 1792-1811, https://doi.org/10.1175/1520-0493(2002)130<1792: CCFTRA $>2.0 . \mathrm{CO} ; 2$.

Read, P. L., 1992: Rotating annulus flows and baroclinic waves. Rotating Fluids in Geophysical and Industrial Applications, E. J. Hopfinger, Ed., Springer, 185-214.

Ricker, W. E., 1954: Stock and recruitment. J. Fish. Res. Board Can., 11, 559-623, https://doi.org/10.1139/f54-039.

Roulston, M. S., and L. A. Smith, 2002: Evaluating probabilistic forecasts using information theory. Mon. Wea. Rev., 130, 1653-1660, https://doi.org/10.1175/1520-0493(2002)130<1653: EPFUIT>2.0.CO;2.

_- and —_, 2003: Combining dynamical and statistical ensembles. Tellus, 55A, 16-30, https://doi.org/10.3402/ tellusa.v55i1.12082.

Silverman, B. W., 1986: Density Estimation for Statistics and Data Analysis. 1st ed. Springer, 175 pp.

Smith, L. A., 1992: Identification and prediction of low dimensional dynamics. Physica D, 58, 50-76, https://doi.org/10.1016/ 0167-2789(92)90101-R.
— H. Du, E. B. Suckling, and F. Niehörster, 2015: Probabilistic skill in ensemble seasonal forecasts. Quart. J. Roy. Meteor. Soc., 141, 1085-1100, https://doi.org/10.1002/qj.2403.

Sprott, J. C., 2003: Chaos and Time-Series Analysis. Oxford University Press, $507 \mathrm{pp}$.

Taylor, K. E., R. J. Stouffer, and G. A. Meehl, 2012: An overview of CMIP5 and the experimental design. Bull. Amer. Meteor. Soc., 93, 485-498, https://doi.org/10.1175/BAMS-D-11-00094.1.

Wang, X., and C. H. Bishop, 2005: Improvement of ensemble reliability with a new dressing kernel. Quart. J. Roy. Meteor. Soc., 131, 965-986, https://doi.org/10.1256/qj.04.120.

Weigel, A. P., M. A. Liniger, and C. Appenzeller, 2008: Can multimodel combination really enhance the prediction skill of probabilistic ensemble forecasts? Quart. J. Roy. Meteor. Soc., 134, 241-260, https://doi.org/10.1002/qj.210.

Weisheimer, A., and Coauthors, 2009: Ensembles: A new multimodel ensemble for seasonal-to-annual predictions-Skill and progress beyond DEMETER in forecasting tropical Pacific SSTs. Geophys. Res. Lett., 36, L21711, https://doi.org/10.1029/ 2009GL040896.

Wilks, D. S., 2006: Comparison of ensemble-MOS methods in the Lorenz 96 setting. Meteor. Appl., 13, 243-256, https://doi.org/ 10.1017/S1350482706002192.

, and T. M. Hamill, 2007: Comparison of ensemble MOS methods using GFS reforecasts. Mon. Wea. Rev., 135, 23792390, https://doi.org/10.1175/MWR3402.1. 